\title{
Detecting C Program Vulnerabilities ${ }^{1}$
}

\author{
Anton Ermakov, Natalia Kushik \\ dept. of Information Technologies \\ Tomsk State University \\ Tomsk, Russsia \\ antonermak@inbox.ru, kushiknatalya@yahoo.com
}

\begin{abstract}
C/C++ language is widely used for developing tools in various applications, in particular, software tools for critical systems are often written in $C$ language. Therefore, the security of such software should be thoroughly tested, i.e., the absence of vulnerabilities has to be confirmed. When detecting $\mathrm{C}$ program vulnerabilities static source code analysis can be used. In this paper, we present a short survey of existing software tools for such analysis and show that for some kinds of $C$ code vulnerabilities this analysis is insufficient. Thus, we briefly present an approach for $S P I N$ based approach for vulnerability detection which may be useful in some cases.
\end{abstract}

Index Terms-C programming language, software vulnerability, static/dynamic detection method

\section{INTRODUCTION}

$\mathrm{T}_{\mathrm{b}}$ HE problem of computer-aided software testing becomes important as the complexity of software tools increases and programs written in $\mathrm{C} / \mathrm{C}++$ programming language are often used in many critical systems. The security of such software should be thoroughly tested, i.e., the absence of vulnerabilities has to be confirmed. There are two different approaches for vulnerability testing: static and dynamic methods. In this paper, we present a short survey of existing tools based on static vulnerability detection methods and show that for detecting some vulnerabilities, for example a buffer overflow vulnerability, SPIN [1] based approach may be more appropriate.

The structure of the paper is as follows. Section II contains preliminaries. Section III is devoted to static code analyzers: a short survey of existing tools for static vulnerability detection is presented in this Section. Section IV discusses a SPIN based approach for vulnerability detection while Section V concludes the paper.

\section{PRELIMINARIES}

A program vulnerability is a property of the program that allows a user to disturb confidentiality, integrity, and/or availability of this software. Given a set of vulnerabilities (features) of a $\mathrm{C}$ program, if the program has none of these

\footnotetext{
${ }^{1}$ This work is partly supported by RFBR-NSC grant № 10-08-92003
}

features then the program is said to be safe w.r.t. the given set of vulnerabilities; otherwise, the program is unsafe w.r.t. this set of vulnerabilities. Vulnerability detection methods can be classified as static and dynamic methods [2]. When static detecting methods are applied the source code is analyzed without running the program while dynamic detection methods require the program of interest to be executed.

Given a $\mathrm{C}$ program, in this paper, when illustrating the approaches, we consider the following types of possible vulnerabilities: type overflow, type conversion overflow, array overflow (incorrect array index), string overflow which can be considered as different types of a buffer overflow vulnerability and double free vulnerability. All these types of vulnerabilities are specified in details in [3]. Type overflow occurs in a $\mathrm{C}$ code when a variable $v$ is defined as a variable of type $t$ and the value $e$ of this variable when executing the code can exceed the maximal value for type $t$. It can occur when a given expression $e$ is assigned to a variable $v$, i.e., the $\mathrm{C}$ code has an instruction $v=e$, and in general, the maximal value for type $t$ might be different for different platforms and operating systems. An array overflow takes place when a programmer deals with an array $a$ that has size_a items while using a variable $a[i]$ for $i>=$ size_a. When analyzing student software tools implementing well-known array algorithms such as different sorts and/or search of minimal/maximal array item, we noticed that many those programs are unsafe w.r.t. type overflow and array overflow (incorrect array index) vulnerabilities. In order to estimate whether existing static methods can detect type overflow and array overflow vulnerabilities we consider three student implementations of array algorithms and run existing tools for detection of such vulnerabilities. In the next section, we present a short survey of existing tools for static code analysis and their outputs for several vulnerable student programs. We then show that some of such vulnerabilities can be detected using SPIN based approach.

\section{STATIC CODE ANALYZERS}

When estimating the security of student implementations of array algorithms we considered the following tasks: calculating the average value of integer array items, the bubble sort, the insertion sort. $\mathrm{C}$ implementations of these programs are specified in the Table 1 which is divided into three 
sections. Table 1.1 contains a $\mathrm{C}$ implementation of calculating the average value of integer array items (Program 1), Table 1.2 contains a $\mathrm{C}$ implementation of the bubble sort (Program 2) while Table 1.3 contains a $\mathrm{C}$ implementation of the insertion sort (Program 3). Program 1 has a type overflow vulnerability in the line

$$
\text { sred+=a[i]; }
$$

There is no check in Program 1 if sred variable value does not exceed the maximal value of the type unsigned short; in this paper, the maximal value equals 65536 and each unsigned short variable occupies two memory bytes. Programs 2 and 3 have an array overflow vulnerability, since array indexes of arrays $a$ and $a r r$ are not checked whether they exceed the number of array items.

\begin{tabular}{|c|c|c|}
\hline \multicolumn{2}{|c|}{$\begin{array}{c}\text { Program } 1- \\
\mathrm{C} \text { implementation of } \\
\text { calculating the average } \\
\text { value of integer array } \\
\text { items }\end{array}$} & $\begin{array}{l}\text { int main()\{ } \\
\text { unsigned short n=0, a[10]; } \\
\text { printf("Enter size of array, } \\
\text { please:"); } \\
\text { scanf("\%d",\&n); } \\
\text { for (int } i=0 ; i<n ; i++) \\
\text { \{printf("\%d. ",i); } \\
\text { scanf("\%d",\&a[i]); } \\
\text { \} } \\
\text { unsigned short sred=0; } \\
\text { for (int i=0; i<n; i++) } \\
\{ \\
\text { sred+=a[i]; } \\
\text { \} } \\
\text { sred/=n; } \\
\text { printf("Sred:\%d",sred); } \\
\text { system("pause"); return sred; } \\
\text { \} }\end{array}$ \\
\hline \multicolumn{3}{|c|}{$\begin{array}{l}\text { Table 1.1 C implementation of array algorithms } \\
\text { (Program 1) }\end{array}$} \\
\hline $\begin{array}{c}\text { Program 2- } \\
\mathrm{C} \text { implementation } \\
\text { of the bubble sort }\end{array}$ & $\begin{array}{l}\text { int } \\
\text { uns } \\
\text { cou } \\
\text { cin } \\
\text { for } \\
\{ \\
\\
\} \\
\text { uns } \\
\text { boo } \\
\text { wh } \\
\{ \\
\text { t }= \\
\text { fo } \\
\{\end{array}$ & 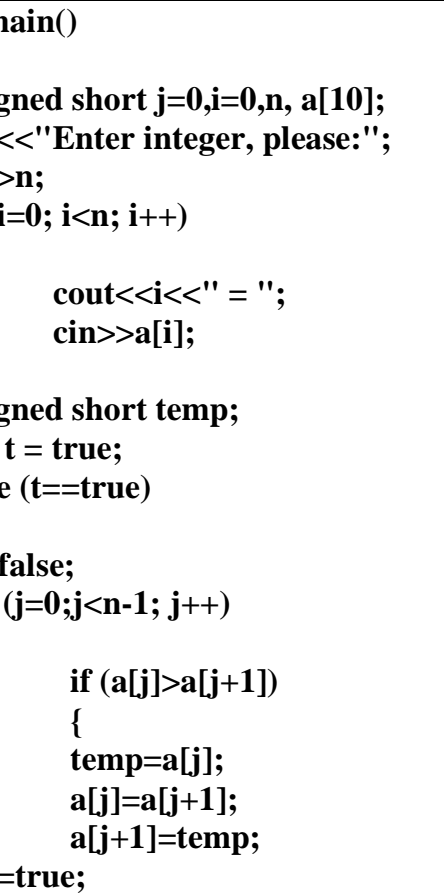 \\
\hline
\end{tabular}

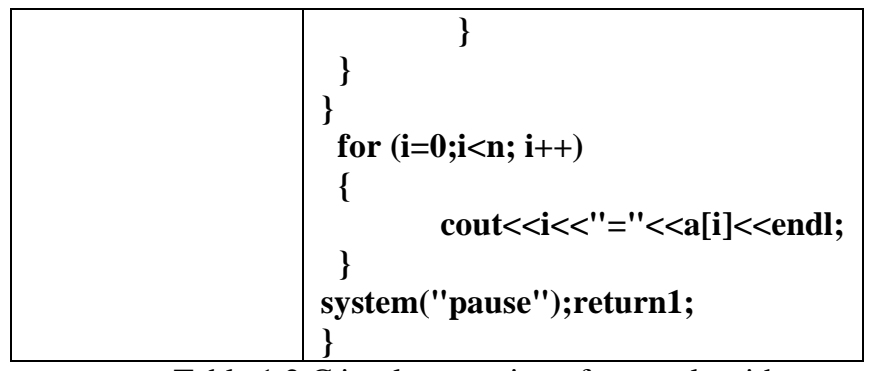

Table 1.2 C implementation of array algorithms (Program 2)

Below we describe the outputs of several static source code analyzers that have been run against $\mathrm{C}$ implementations in the Table 1.

A. ITS4 is a static code analyzer that has been developed in USA by the Cigital company in 1992 [4]. The ITS4 is a tool for static detection vulnerabilities in $\mathrm{C} / \mathrm{C}++$ programs. The tool can be executed under Windows or Linux operating systems.

\begin{tabular}{|c|c|}
\hline $\begin{array}{c}\text { Program } 3- \\
\text { C implementation } \\
\text { of the insertion sort }\end{array}$ & 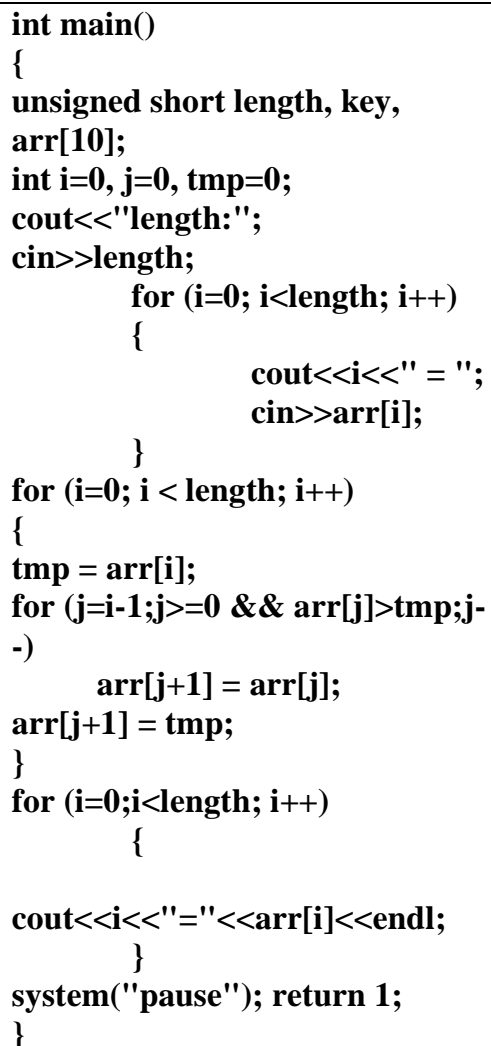 \\
\hline
\end{tabular}

When analyzing a given C code the ITS4 relies on its database of potentially dangerous $\mathrm{C}$ functions and if there is a call for such dangerous function in the given code the ITS4 returns a corresponding report with some recommendations about proposes (preferable changes) in the code. The ITS4 tool is a free software tool that can be easily downloaded from web-site [4]. We executed ITS4 against Programs 1, 2, 3 (Tables 1.1, 1.2, 1.3) and the ITS4 has detected two calls for 
dangerous functions. Those are $\operatorname{scanf}()$ and $\operatorname{printf}()$, in particular, the ITS4 has reported that $\operatorname{scanf(})$ is a function of a high risk for a buffer overflow vulnerability.

B. Flawfinder is also a static $\mathrm{C} / \mathrm{C}++$ code analyzer that has been developed by David A. Wheeler in May, 2004 [5]. Flawfinder "scans" a given code and similar to the ITS4, has a list of potentially dangerous instructions of a code. Given a code, selected dangerous instructions (if any) are then ordered according to the risks. The Flawfinder report for a programmer points out the calls for dangerous functions and proposes a way for changing the code. However for the above Programs 1, 2, 3 the Flawfinder report has only one dangerous function - system () and the recommendation "try using a library call that implements the same functionality if available".

C. Graudit is a tool that can also help to statically detect several C code vulnerabilities [6]. In order to run this tool it is necessary to call utility Grep under Unix operating system. As usual, there can be several options how to run this utility but in the simplest case only the path to cpp file has to be specified. As a result, a colorful report will appear where for a given $\mathrm{C}$ program, some dangerous instructions are blue colored. One can also manually add more instructions into the database of dangerous functions. For each program in Table 1 the Graudit colored functions scanf(), printf() and stream input/output operators cin and cout.

D. CppCheck 1.46 is a tool with the original name $C++$ check that has been developed by Daniel Marjamäki and Cppcheck team from 2007 until 2010 [7]. The CppCheck utility is specialized for memory leakage vulnerabilities. As it is mentioned in [7] CppCheck has detected 21 errors in the Linux Core and many other errors in free software. The Cppcheck is also a free software tool under the conditions of the GNU General Public License. We have run the Cppcheck against above Programs 1, 2, 3 and the output message "No errors found" has been returned.

E. AEGIS is another tool for static detection vulnerabilities in $\mathrm{C} / \mathrm{C}++$ programs [8]. The AEGIS has been developing in Digitek Labs since 2008. This laboratory is strongly connected with Saint-Petersburg Polytechnic University, Russia. One of the advantages of this tool is that the AEGIS supports vulnerability detection for several files simultaneously if they are united in one project. The AEGIS detects vulnerabilities that can often occur in $\mathrm{C}$ programs, such as memory leakage, incorrect pointers, incorrect array indexes, uninitialized variables, the use of potentially dangerous functions etc. In order to statically detect these vulnerabilities the AEGIS derives the abstract model of the program for verification. The free usage of the analyzer is available via the official Digitek Labs web-site [9]. Before running this tool it is necessary to make some transformations of a given $\mathrm{C}$ code for further compiling. For example, in the $A E G I S$, it is prohibited to analyze a code where two or more $\mathrm{C}$ instructions are located in the same program line. We have correspondingly changed the above Programs 1, 2, 3 and have run the AEGIS. For Program 1 of average value calculating the $A E G I S$ detected an incorrect array index for the array arr while for Programs 2 and 3 of array sorts the AEGIS mentioned only the call of unsafe function system().
F. There are other static code analyzers that can be used for vulnerability detection in $\mathrm{C}$ programs. For example, Cqual [10], developed by Dan Wilkerson in 2004, Eshelon AK-VS [11] developed in Russia, Klocwork Truepath [12] developed by Klocwork company and Coverity Static Analysis [13] developed by Coverity company in USA, MOPS [14] and $B O O N$ [15] are tools for static detection vulnerabilities. We could not execute these tools due to some reasons such as a high price, lack of documentation, absence of demonstrating version etc. However, according to their descriptions [10-15], all these tools are developed for static detection of vulnerabilities and many of them allow static analysis not only for $\mathrm{C} / \mathrm{C}++$ code but also for Java or $\mathrm{C} \#$ programs.

According to the above short survey of static code analyzers, one can conclude that most existing tools only search for dangerous functions and despite of their descriptions do not detect type overflow and incorrect array index vulnerabilities. The latter means that for some kinds of software vulnerabilities static detection is not enough, that is the reason why in the next section we present a brief overview of an approach for dynamic detection vulnerabilities [3].

\section{SPIN BASED APPROACH FOR DETECTING VULNERABILITIES}

Most existing tools providing dynamic detection vulnerabilities are based on randomly generated input data for a given program. Thus, it is difficult to guarantee the fault coverage for such security testing. There also exist special tools for distributed programs testing, for example, Helgrind [16] that is designed for multithreaded programs testing. We note that this tool does not support buffer overflow detection technique but it is able to control synchronization between threads.

There are other model checking techniques which are widely used for vulnerability detection. Working together with our French colleagues we proposed a detection technique based on SPIN model checker $[1]^{2}$ and have partially presented the obtained results in the technical report [3]. In this case, a vulnerability is described as a property that has to be verified. However, SPIN accepts a program written in PROMELA language and thus, the first question is how to translate a $\mathrm{C}$ code into PROMELA instructions when verifying a property of interest. If the program is vulnerable, i.e., possesses a "bad" feature, then SPIN produces a counterexample that corresponds to the values of internal variables or of input data of the program. We note that, according to SPIN documentation features might be specified as temporal logic formulas or Buchi automata [17]. In the former case, we propose how to inject such data into the program in order to show a programmer which part of the code is vulnerable. The proposed technique somehow takes into account both static and dynamic vulnerability detection, since PROMELA model is verified statically while counterexample is injected into the program through its runtime. In [3], some discussions can be found how to translate $\mathrm{C}$ instructions into PROMELA instructions and how the

\footnotetext{
${ }^{2}$ The work was done together with French scientific group of Prof. Ana Cavalli (TELECOM \& Management Sud Paris)
} 
injection procedure can be implemented. In PROMELA language verified properties are described as assertions and such assertions have to be constructed for each type of vulnerabilities. Unfortunately the translation performed by MODEX tool [18] cannot be applied directly and since we are in the process of developing new automatic tools for such translation, some $\mathrm{C}$ codes were manually converted into PROMELA codes and corresponding assertions were added. We have applied a proposed technique to the above Programs $1,2,3$ and SPIN produced counterexamples for all of them. We injected data according to these counterexamples, found out that the programs return wrong results and no error message about "bad" input data has appeared, i.e., SPIN has detected type overflow and array overflow vulnerabilities in the above programs. For example, for Program 1 a counterexample produced by SPIN has the value 10005 for each array item value, the returned result when running the program was 3451 while the right value should be 100050 , i.e., this $\mathrm{C}$ code has a type overflow vulnerability.

For Program 2 SPIN produced a counterexample as well as for the array dimension as for array item value. In this case when detecting array overflow vulnerability the counterexample was $n=11$ when each array item equals 11 too. When detecting type overflow vulnerability SPIN produced the value 70035 that was then assigned to each array item. After applying these input data to Program 2 incorrect result has been obtained when running the $\mathrm{C}$ program while no error occurred. According to the incorrect result that can easily be checked, one can conclude that SPIN has detected type and array overflow in Program 2. For Program 3 (Table 1) SPIN has produced the same counterexample $n=11$ for an array overflow while in the counterexample for a type overflow vulnerability, each array item was assigned to 80040 .

In order to compare SPIN based vulnerability detection technique with other tools providing dynamic vulnerability detection we have run the Memcheck utility of Valgrind software [15] against Programs 1, 2, 3. Memcheck is designed to detect memory leakages in $\mathrm{C} / \mathrm{C}++$ programs and incorrect use of uninitialized values. Valgrind allows a programmer to assign desirable values to input variables and by use of a virtual machine the Memcheck utility checks whether memory leakage occurs during the program execution. We have run Memcheck against Programs 1, 2, 3 with counterexamples produced by SPIN and neither type overflow nor array overflow vulnerability has been mentioned.

Based on the obtained experimental results, we can conclude that SPIN based detection techniques could be useful when analyzing the $\mathrm{C}$ code safety.

\section{CONCLUSIONS}

In this paper, we have presented a short survey of existing tools providing vulnerability detection in $\mathrm{C} / \mathrm{C}++$ programs. Several tools have been executed against student implementations of array algorithms. The experimental results clearly show that for some kinds of $\mathrm{C}$ code vulnerabilities static analysis can be insufficient and we have presented a brief overview of a SPIN-based approach for vulnerability detection. The obtained preliminary results clearly show that SPIN based detection techniques could be useful when analyzing the $\mathrm{C}$ code safety. In this paper, we did not discuss vulnerability detection techniques based on other model checkers; such a comparison is a part of our future work.

\section{REFERENCES}

[1] G. Holzmann. Spin Model Checker. Primer and Reference Manual. Addison Wesley, 2003.

[2] Willy Jimenez, Amel Mammar, and Ana R. Cavalli. Software Vulnerabilities, Prevention and Detection Methods. A Review, SECMDA workshop.- Enschede, The Netherlands, June, 24, 2009.

[3] Technical report of the joint FCP Russian-French grant № 02.514.12.4002, Step 4 .

[4] Cigital [Electronic resource] - http://www.cigital.com/its4/

[5] Flawfinder home page [Electronic resource] http://www.dwheeler.com/flawfinder

[6] Just Another Hacker [Electronic resource] http://www.justanotherhacker.com/projects/graudit/download.html

[7] Sound Forge [Electronic resource] http://sourceforge.net/apps/mediawiki/cppcheck/

[8] Digitek Labs [Electronic resource] - http://www.digiteklabs.ru/aegis/

[9] Digitek Labs [Electronic resource] - http://aegisdemo.digiteklabs.ru/s2a.webserver/

[10] Department of computer science. University of Maryland [Electronic resource] - http://www.cs.umd.edu/ jfoster/cqual/

[11] Soft Line [Electronic resource] - http://soft.softline.ru/NPOEchelon/eshelon-ak-vs/

[12] Klocwork [Electronic resource] http://www.klocwork.com/products/insight/klocwork-truepath/

[13] Coverity [Electronic resource] http://www.coverity.com/products/static-analysis.html

[14] Electrical engineering and computer sciences [Electronic resource] http://www.cs.berkeley.edu/ daw/mops/

[15] Electrical engineering and computer sciences [Electronic resource] http://www.cs.berkeley.edu/ daw/boon/

[16] Valgrind [Electronic resource] - http://valgrind.org/info/tools.html

[17] SPIN [Electronic resource] - http://spinroot.com/

[18] Modex [Electronic resource] - $\mathrm{http} / / \mathrm{cm}$.belllabs.com $/ \mathrm{cm} / \mathrm{cs} /$ what $/$ modex/index.html 